\title{
BLER Performance Evaluation of LTE Device-to-Device Communications
}

Jian Wang

Richard Rouil

This publication is available free of charge from: https://doi.org/10.6028/NIST.IR.8157 


\title{
BLER Performance Evaluation of LTE Device-to-Device Communications
}

\author{
Jian Wang \\ Richard Rouil \\ Wireless Networks Division \\ Communications Technology Laboratory
}

This publication is available free of charge from:

https://doi.org/10.6028/NIST.IR.8157

November 2016

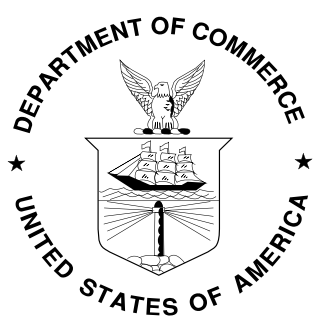

U.S. Department of Commerce Penny Pritzker, Secretary

National Institute of Standards and Technology Willie May, Under Secretary of Commerce for Standards and Technology and Director 


\begin{abstract}
Long-Term Evolution (LTE) supporting Device-to-Device (D2D) communication is a feature introduced in $3^{\text {rd }}$ Generation Partnership Project (3GPP) release 12. D2D communication is critical in public safety applications, which enables communication among first responders when the network infrastructure may not be available. In order to characterize the performance of D2D communications using network simulation tool (e.g. ns-3), accurate physical layer error model is important. In this document, we present our D2D physical channel simulations to study the BLock Error Rate (BLER) performance of the physical channels. We also demonstrate how to integrate the simulation results into network simulator to perform system level simulation.
\end{abstract}

\title{
Table of Contents
}

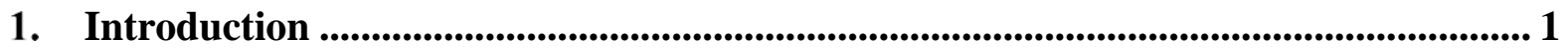

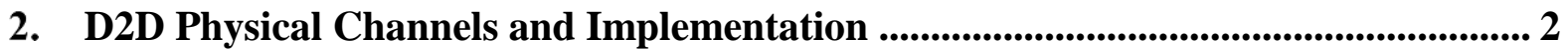

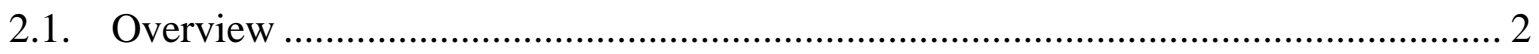

2.2. Physical Sidelink Control Channel (PSCCH) ................................................. 2

2.3. Physical Sidelink Broadcast Channel (PSBCH) .................................................. 5

2.4. Physical Sidelink Discovery Channel (PSDCH) ............................................... 7

2.5. Physical Sidelink Shared Channel (PSSCH) .................................................. 9

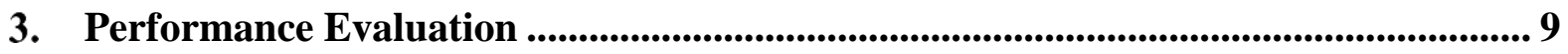

4. Incorporating D2D Physical Channel BLER Performance in Network Simulator 13

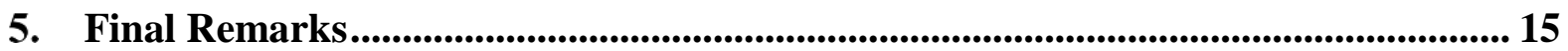

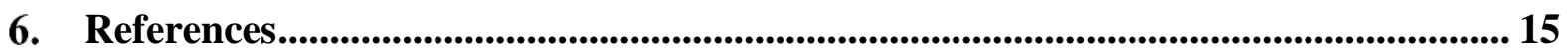




\section{Introduction}

Long-Term Evolution (LTE) supporting Device-to-Device (D2D) communication is a feature introduced in $3^{\text {rd }}$ Generation Partnership Project (3GPP) release 12 [1]. Via D2D communications, devices can directly communicate with each other without the aid of cellular network infrastructure [2]. D2D communication is critical in public safety communications, which enables communication among first responders when the network infrastructure is not available.

In order to characterize the performance of D2D communications on various network settings, network simulation tools have been developed and utilized. For example, ns-3 [2] is one of known open source discrete-event network simulation tools that can be used to evaluate the performance of different network architectures, protocols, and applications (for example, public safety) with respect to throughput, end-to-end delay, and other network performance metrics. As an essential component in ns-3, the physical layer model plays two important roles. First, the physical layer model can be used to simulate whether a transport block, which is referred to as the data received by the physical layer from the upper layer, can be transmitted successfully over the physical channel. Second, the physical layer model can be used by the scheduler to maximize spectral efficiency by adapting the channel Modulation and Coding Schemes (MCS) used to transmit the data.

In the latest ns-3 release ns-3.25, a physical layer error model designed for LTE physical channels, such as Physical Downlink Shared CHannel (PDSCH) and Physical Downlink Control CHannel (PDCCH), can be used to assess the performance of the LTE system. As error models for D2D physical channels are not available in the latest ns-3 release, the objective of this study is to bridge this gap.

In this study, we simulate several D2D channels using and extending the MATLAB LTE Toolbox $^{1}$ [3], including Physical Sidelink Control Channel (PSCCH), Physical Sidelink Broadcast Channel (PSBCH), Physical Sidelink Discovery Channel (PSDCH), and Physical Sidelink Shared Channel (PSSCH). For the PSCCH and PSBCH, we investigate the relationship between BLock Error Rate (BLER) and Signal to Noise Ratio (SNR). For the PSDCH, we study the relationship between BLER and SNR with respect to each Hybrid Automatic Repeat reQuest (HARQ) transmission. For the PSSCH, we investigate the relationship between BLER and SNR with respect to different Modulation and Coding Scheme (MCS) and for each HARQ transmission. In 3GPP specifications release 12, physical broadcast services are used for D2D communications in LTE systems, and there is no feedback for each HARQ transmission. Thus, a sender always sends four Redundant Versions (RVs) of data to a receiver, which contains different combinations of information bits and error protection bits.

The remainder of this report is as follows: In Section 2, we provide an overview of our simulation and describe the implementation of the four D2D physical channels in detail. In

${ }^{1}$ Certain commercial equipment, instruments, or materials are identified in this report in order to specify the experimental procedure adequately. Such identification is not intended to imply recommendation or endorsement by the National Institute of Standards and Technology, nor is it intended to imply that the materials or equipment identified are necessarily the best available for the purpose. 
Section 3, we present the simulation results. In Section 4, we discuss how to incorporate the D2D physical channel BLER performance data in the system level simulation tool. In Section 5, we conclude the report and give the final remarks.

\section{D2D Physical Channels and Implementation}

In this section, we first give an overview and then present the detailed design and implementation of LTE-based D2D channels.

\subsection{Overview}

We leverage the MATLAB LTE Toolbox to implement D2D physical channels and characterize the performance of these channels. The MATLAB LTE toolbox is an add-on component of MATLAB, which provides standard-compliant functions and applications for the design and development of LTE systems [3]. The toolbox can be used to support the performance assessment of physical layer development, verify standards development, or generate standard-compliant LTE waveforms. By using the MATLAB LTE toolbox, we can configure, simulate, and measure communication links, and investigate the performance of various physical channels, with respect to BLER versus SNR, when different MCSs are used. Notice that BLER is referred to as the ratio of the number of erroneous blocks to the total number of blocks received over the wireless channel.

For the system level simulation, different channel models (the large-scale propagation model, small-scale fading model, etc.) should be considered. Because, in ns-3, those channel models can be implemented as a separate component (building model, fading model, etc.) [4], the channel that we consider in our simulation is an Additive White Gaussian Noise (AWGN) channel. In addition, multiple transmit and receive antennas should be considered in the system level simulation. The performance gain that a Multiple Input Multiple Output (MIMO) scheme provides to the system, in comparison with Single Input Single Output (SISO), can be approximated using the MIMO model in ns-3 [4]. Therefore, in our simulation, we consider SISO only.

In order to evaluate the channel's performance with respect to BLER versus SNR, we study the LTE-based D2D channels, and implement them using the MATLAB LTE toolbox. We implement the key communication modules in both transmitter and receiver, including channel coding/decoding, resource mapping/demapping, QPSK/16QAM modulation/demodulation, scrambling/descrambling and SC-FDMA modulation and demodulation. We vary the SNR of the transmitted signal by changing the power of noise, and derive the BLER once the transport block is recovered after passing through the transmission and receiving process chain. In the following, we present the implementation of individual LTE D2D channels in detail.

\subsection{Physical Sidelink Control Channel (PSCCH)}

In LTE, PSCCH is used to transmit Sidelink Control Information (SCI) message, which is used by a receiver to decode the physical sidelink shared channel (denoted as PSSCH). Note that PSSCH is a data channel in sidelink communication. An SCI message contains the control information, including the shared channel resource allocation, modulation and coding schemes, time resource pattern, group destination identifier, and frequency hopping flag. To ensure the reliability of the SCI message delivery, each message is transmitted in 
two identical instances, where each instance occupies one Resource Block (RB). The two instances are transmitted over two different subframes.

Table 1: PSCCH Reference Channels [5]

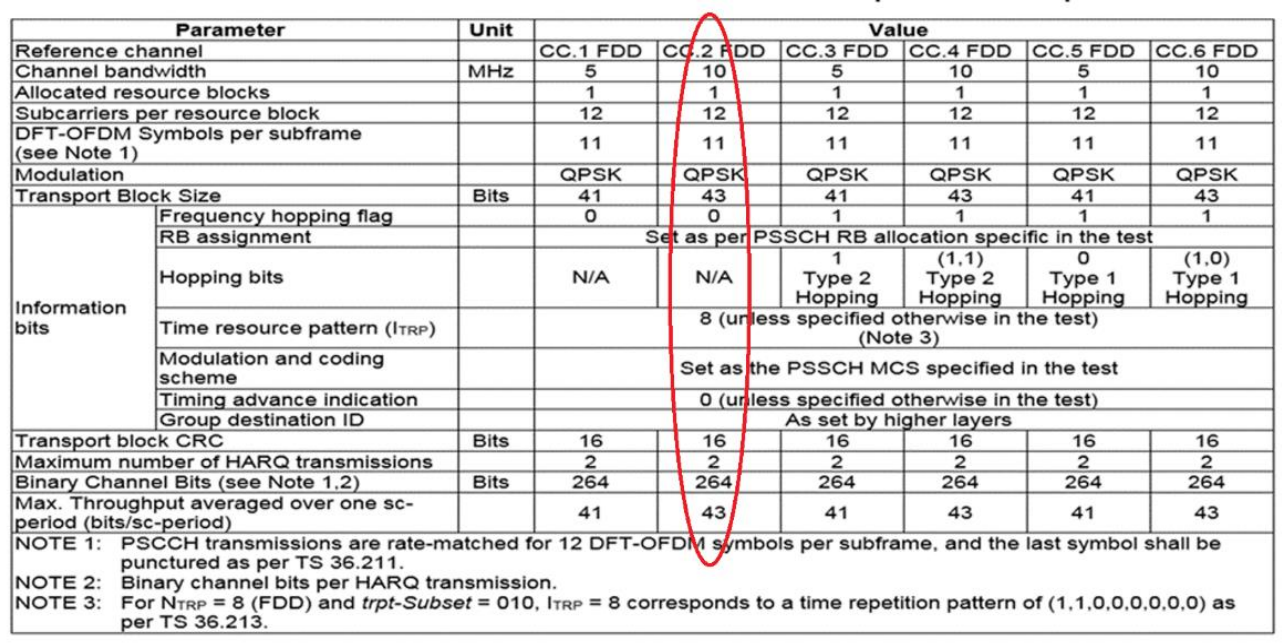

As a control channel for LTE D2D communication, 3GPP 36.101 [5] defines several reference channels for PSCCH, as shown in Table 1. As we can see from the table, all reference channels have the most common setting (allocated resource blocks of 1, DFTOFDM symbols per subframe of 11, modulation mechanism using Quadrature Phase Shift Keying (QPSK), transport block Cyclic Redundancy Check (CRC) of 16, etc.). In our implementation, we choose the reference channel CC.2 FDD because it matches our system simulation configuration well. Notice that our design and implementation of LTE-based D2D channel models is generic and other references channels can be easily extended.

The basic workflow of our PSCCH implementation is illustrated in Figure 1. On the left side of the figure, we show the modules in the transmitter. The modules in the receiver are shown on the right side of the figure. At the transmitter side, 43 bits of SCI is received at the physical layer from the upper layer. Notice that the channel coding for SCI follows the coding mechanism used by the Downlink Control Information (DCI) of LTE physical downlink control channel. However, in PSCCH, the scrambling is not used during the CRC attachment because the receiver involved in D2D communication may not always know the transmitter's identity (ID). The channel coding uses a 1/3 tail-biting convolutional code. In addition, rate matching is used to fit the output bits into a $\mathrm{PSCCH}$ resource grid.

After channel coding and rate matching, LTE uplink interleaving is applied to the coded bits to combat burst error occurring during the transmission. Notice that in D2D uplink interleaving, only the data is applied. Before modulation, the bits go through a bit-level scrambling process by conducting modulo 2 addition with a pseudo random scrambling sequence. For PSCCH, the scrambling sequence is generated using a fixed initial value of 510. By doing so, the receiver can decode the SCI message without the knowledge of the transmitter's ID. QPSK modulation is then used to convert the scrambled bits to the symbols of complex value. Next, layer mapping is used. Notice that, in our case, the layer 
mapping is simple, because a single antenna port is used according to [6]. Similar to LTE Uplink, Single Carrier Frequency Division Multiple Access (SC-FDMA) is used in D2D communication to reduce signal peak-to-average power ratio and improve the efficiency of the power amplifier in User Equipment (UE). In order to implement SC-FDMA, the LTE uplink Discrete Fourier Transform (DFT) precoding is applied before resource mapping.
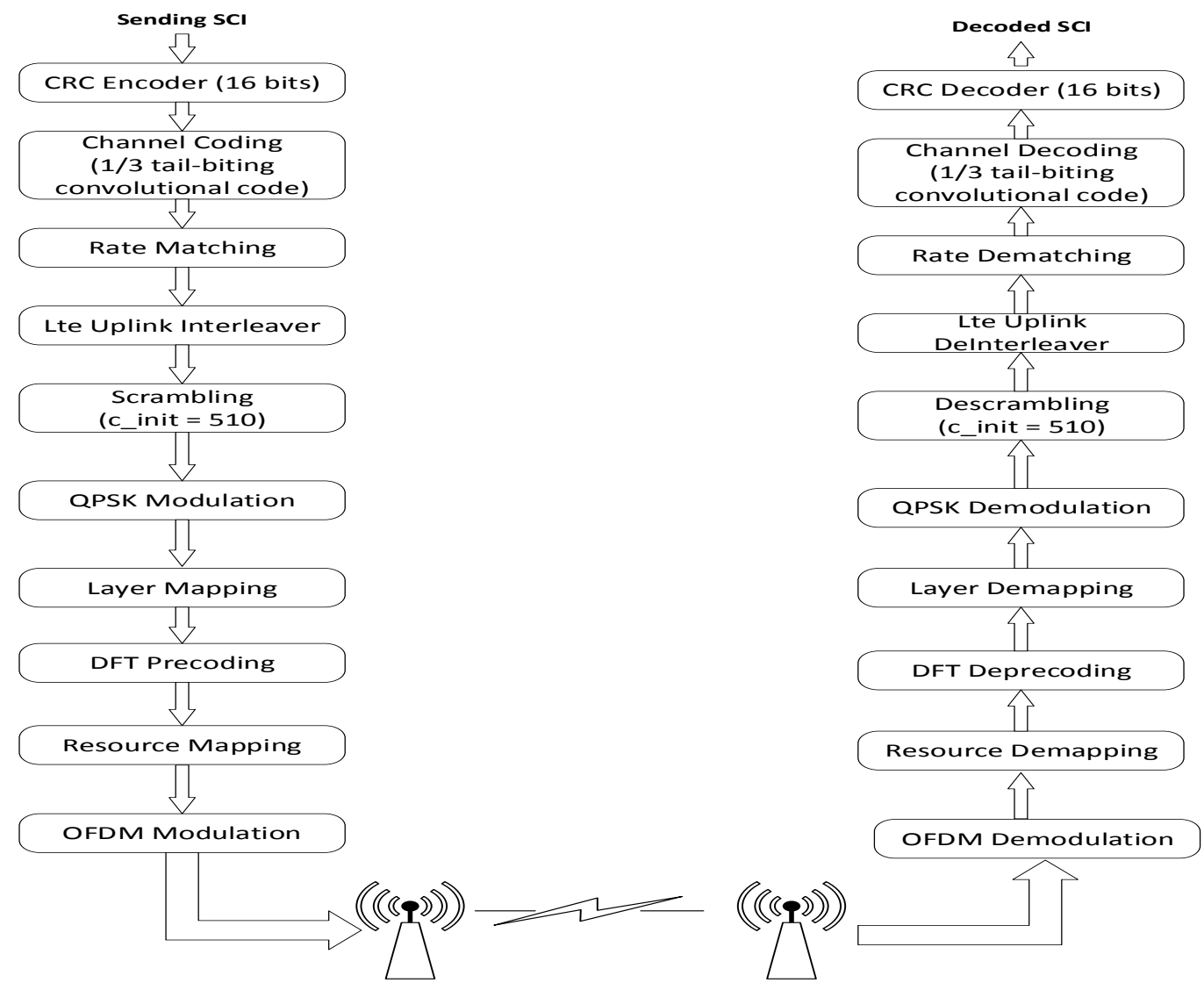

Figure 1: Workflow of PSCCH

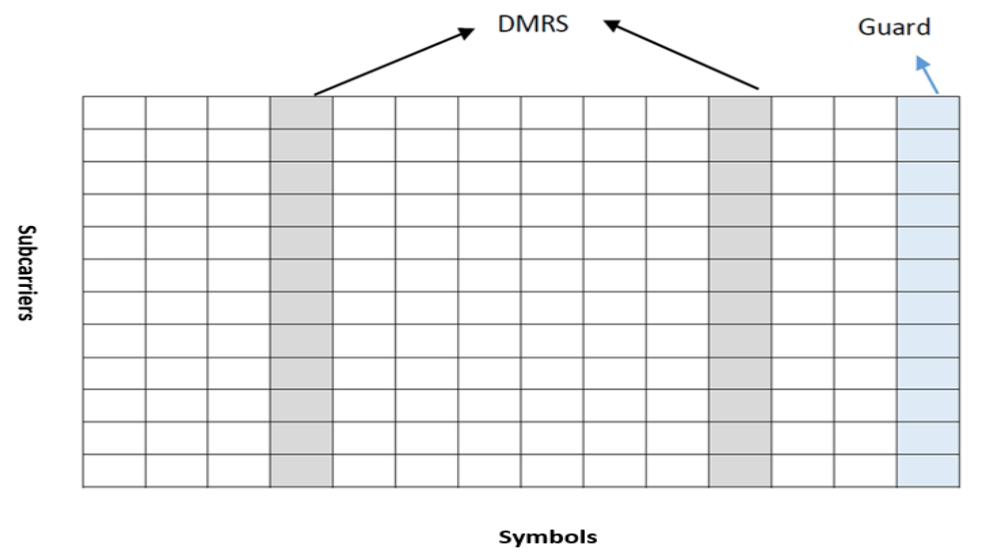

Figure 2: Resource Grid of PSCCH 
The next step is to fill these DFT pre-coded symbols into a PSCCH resource grid, shown in Figure 2, and convert a serial stream to a parallel stream. As shown in the figure, where symbols use normal cyclic prefix, one PSCCH transport block occupies one Physical Resource Block (PRB), which consists of 12 subcarriers. For each subcarrier, the fourth and eleventh elements are reserved for DEmodulation Reference Signal (DMRS), which can be used for channel estimation and facilitating coherent modulation. The last resource element of each subcarrier is punctured to serve as a guard period. The guard period helps resolve the possible collision between UE's receiving signal associated with D2D communication and UE's LTE uplink signal, because accurate timing advance information may not be available in D2D communication. The parallel stream will finally go through the OFDM modulation and be converted to a time-domain signal to be transmitted over the wireless channel.

On the receiver side, a reverse processing is applied to the received signal. First, OFDM demodulation is applied to convert the signal from the time domain back to the frequency domain. Then, a series of PSCCH symbols are selected from the PSCCH resource element grid via resource demapping, followed by Inverse DFT deprecoding, layer demapping, and QPSK demodulation. After that, the demodulated bit stream goes through descrambling, deinterleaving, and DCI decoding to recover the transmitted SCI. The results of CRC error checking can determine whether the transport block is transmitted correctly or not.

\subsection{Physical Sidelink Broadcast Channel (PSBCH)}

The PSBCH is used to carry SideLink Broadcast Channel (SL-BCH), which is a transport channel used to transmit logic channel information of Sidelink Broadcast Control Channel $(\mathrm{SBCCH})$. The $\mathrm{SBCCH}$ is mainly used to broadcast system level information, including system bandwidth, D2D frame number, etc. 3GPP Spec. 36.101 [5] defines a reference channel for PSBCH. As shown in Table 2, the reference channel is CC.1 FDD, the channel bandwidth is set to be either $5 \mathrm{MHz}$ or $10 \mathrm{MHz}$, the allocated number of resource blocks is 6, the modulation scheme is set to QPSK, and 16 bit CRC protection is used. In addition, the transport block size is 40 bits and the number of binary channel bits becomes 1008 after channel coding and bit mapping.

Table 2: PSBCH Reference Channels [5]

\begin{tabular}{|l|c|c|}
\hline \multicolumn{1}{|c|}{ Parameter } & Unit & Value \\
\hline Reference channel & & CP.1 FDD \\
\hline Channel bandwidth & $\mathrm{MHz}$ & $5 / 10$ \\
\hline Allocated resource blocks & & 6 \\
\hline Subcarriers per resource block & & 12 \\
\hline $\begin{array}{l}\text { DFT-OFDM Symbols per subframe } \\
\text { (see Note 1) }\end{array}$ & & 7 \\
\hline Modulation & & QPSK \\
\hline Transport Block Size & Bits & 40 \\
\hline Transport block CRC & & 16 \\
\hline Maximum number of HARQ transmissions & Bits & 1008 \\
\hline Binary Channel Bits (see Note 1,2) & kbps & 1 \\
\hline Max. Throughput averaged over 40ms & & 1 \\
\hline NOTE 1: PSBCH transmissions are rate-matched for 8 DFT-OFDM symbols per \\
subframe, and the last symbol shall be punctured as per TS 36.211.
\end{tabular}


The basic workflow of our implementation on PSBCH is shown in Figure 3. On the left side of the figure, we show the transmitter modules, while the receiver modules are shown on the right side of the figure.

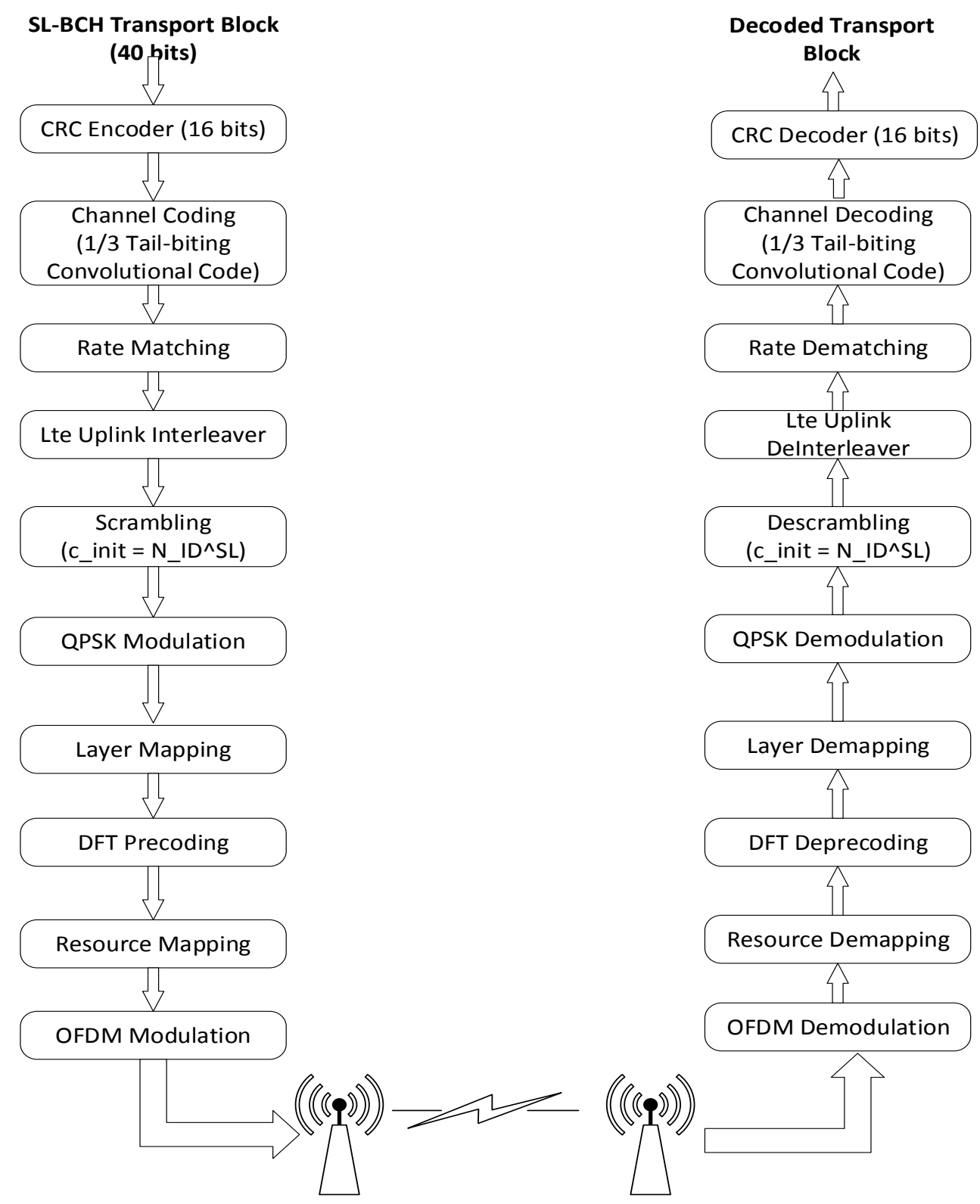

Figure 3: Workflow of PSBCH

At the transmitter side, 40 bits of transport block is received at the physical layer from the upper layer transport channel (SL-BCH). The SL-BCH transport block is first given CRC error checking and goes through a coding rate $1 / 3$ tail biting convolution code with a constraint length of 7 for error protection. Then, rate matching is applied to the channel coding output similar to PSCCH processing shown in Figure 1. These bits then pass through Physical Uplink Shared CHannel (PUSCH) interleaving and are further scrambled by a scrambling sequence. The scrambling generator is initiated as the Sidelink channel 
$\mathrm{ID}, c_{\mathrm{init}}=N_{\mathrm{ID}}^{\mathrm{SL}}$ [7], which corresponds to the physical cell ID when the UE is in cell coverage, or synchronization source ID when the UE is out of coverage. The modulation scheme used by PSBCH is QPSK modulation, and DFT precoding is applied before resource mapping to use SC-FDMA. After the resource mapping, OFDM modulation is applied.

On the receiver side, reverse processing is applied to the received signal. First, OFDM demodulation is applied to convert time-domain signal back to frequency-domain. Then, a series of PSBCH symbols are selected from a PSBCH resource element grid via resource demapping, followed by Inverse DFT deprecoding, layer demapping, and QPSK demodulation. After that, the demodulated bit stream goes through descrambling, deinterleaving, and a coding rate $1 / 3$ tail biting convolution decoding to recover the transmitted transport block. The results of CRC error checking are applied to determine whether the transport block is transmitted correctly or not via the channel.

Notice that in the resource mapping of PSBCH, PBCCH occupies the central 72 subcarriers and 7 SC-OFDM symbols within a subframe $(1 \mathrm{~ms})$. The resource element grid of PSBCH is shown in Figure 4. Along with PSBCH in the resource grid, there exist the Primary Sidelink Synchronization Signal (PSSS) and Secondary Sidelink Schronization Signal (SSSS), where each occupies the center 62 subcarriers and 2 symbols in the $1 \mathrm{~ms}$ subframe. The synchronization signal is used for other UEs to synchronize with broadcasting UE and decode PSBCH broadcasting information. Similar to PSCCH, PSBCH resource elements also contain 2 DMRS symbols per subcarrier in a subframe, and the last symbol in a subcarrier is punctured to serve as guard period.

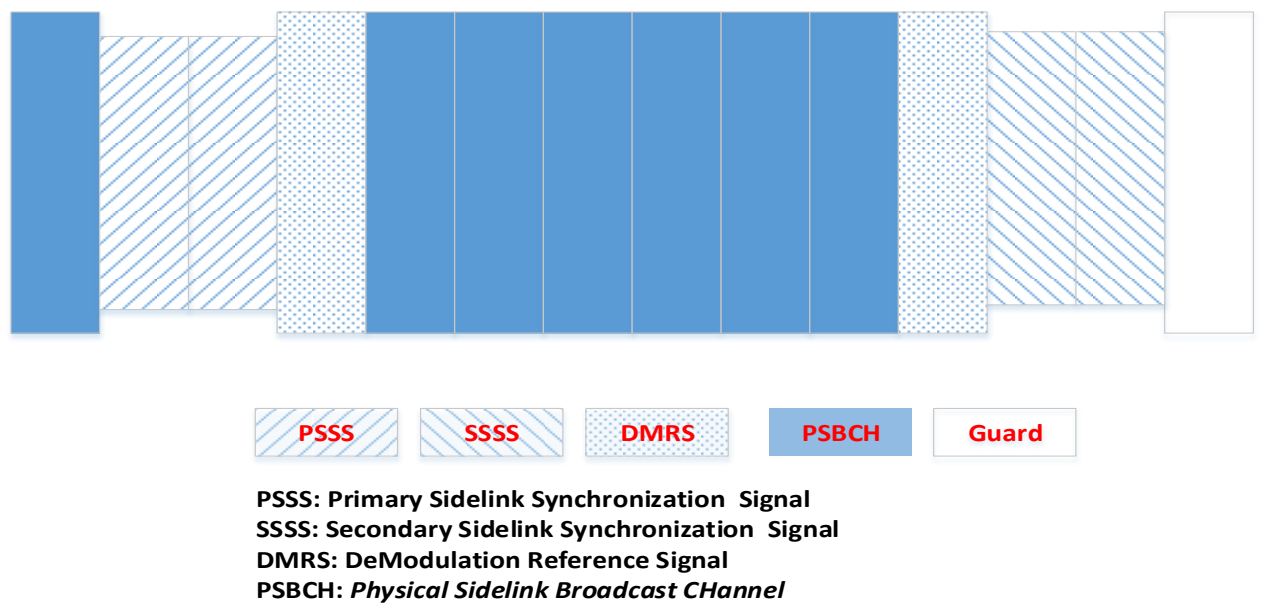

Figure 4: Resource Element Grid of PSBCH

\subsection{Physical Sidelink Discovery Channel (PSDCH)}

PSDCH is used to carry discovery information in LTE D2D communication. With discovery service, a UE is able to identify other UEs in the proximity. PSDCH occupies two resource blocks, and has a fixed transport block size of 232 bits. Each transport block will be transmitted up to four times (4 HARQs), as no physical layer feedback is available, and the receiver will soft combine the transmissions to conduct decoding. The number of transmissions is provided by the eNodeB if the UE is in coverage, or preconfigured if the $\mathrm{UE}$ is out of coverage. 
At the transmitter, similar to LTE PUSCH processing, the transport block is first attached a 24 bit CRC for error detection. Then, Turbo coding is applied to the entire block, followed by rate matching. Next, PUSCH interleaving is applied without any control information. After the interleaving, PUSCH scrambling is used to scramble the bit stream for one transport block. The scrambling sequence is generated using seed $C_{\text {init }}=510$, similar to PSSCH, and QPSK is the modulation scheme used to convert the bit streams to symbols. Again, only a single antenna port is used on the PSDCH transmission in our implementation, so all the symbols will simply be mapped to one layer. SC-FDMA precoding, resource grid mapping, and OFDM modulation will be applied in the same way as PUSCH.

Due to the similarity between PSDCH and PUSCH, we leverage the MATLAB LTE toolbox, using PUSCH simulation results to approximate PSDCH. In PSDCH, QPSK is selected as the modulation scheme, which is the same as the modulation scheme used in PUSCH MCS0-9. In addition, PSDCH uses the same channel coding algorithm as PUSCH, while a different coding rate is used. In order to approximate PSDCH performance, we use the PUSCH MCS error curve that has the closest coding rate as PSDCH. The PSDCH coding rate, denoted by $C R_{P S D C H}$, can be derived by

$$
\begin{aligned}
C R_{P S D C H}= & \frac{N_{T B}+N_{C R C}}{N_{R B} \times N_{S C}^{R B} \times N_{\text {sym }}^{S C} \times N_{\text {bits }}^{\text {sym }}}=\frac{232+24}{2 \times 12 \times 11 \times 2} \\
& =0.48
\end{aligned}
$$

Here, $N_{T B}$ is the transport block size, which has a fixed value of 232 in this case, $N_{C R C}$ is the number of CRC bits, $N_{R B}$ is the number of resource blocks for PSDCH, which is fixed to be $2, N_{S C}^{R B}$ is the number of subcarriers in a resource block, $N_{s y m}^{S C}$ is the number of PSDCH symbols carried per subcarrier, and $N_{\text {bits }}^{\text {sym }}$ represents the number of bits carried in a QPSK symbols.

\begin{tabular}{|c|c|c|c|c|c|c|c|c|c|c|}
\hline \multirow{2}{*}{$I_{\mathrm{TBS}}$} & \multicolumn{10}{|c|}{$N_{\text {PRB }}$} \\
\hline & 1 & 2 & 3 & 4 & 5 & 6 & 7 & 8 & 9 & 10 \\
\hline 0 & 16 & 32 & 56 & 88 & 120 & 152 & 176 & 208 & 224 & 256 \\
\hline 1 & 24 & 56 & 88 & 144 & 176 & 208 & 224 & 256 & 328 & 344 \\
\hline 2 & 32 & 72 & 144 & 176 & 208 & 256 & 296 & 328 & 376 & 424 \\
\hline 3 & 40 & 104 & 176 & 208 & 256 & 328 & 392 & 440 & 504 & 568 \\
\hline 4 & 56 & 120 & 208 & 256 & 328 & 408 & 488 & 552 & 632 & 696 \\
\hline 5 & 72 & 144 & 224 & 328 & 424 & 504 & 600 & 680 & 776 & 872 \\
\hline 6 & 328 & 176 & 256 & 392 & 504 & 600 & 712 & 808 & 936 & 1032 \\
\hline 7 & 104 & 224 & 328 & 472 & 584 & 712 & 840 & 968 & 1096 & 1224 \\
\hline 8 & 120 & (256) & 392 & 536 & 680 & 808 & 968 & 1096 & 1256 & 1384 \\
\hline
\end{tabular}

Table 3: Transport Block Size Table (Dimension 34x110) [Table 7.1.72.1-1 in [8]]

To approximate PSDCH using PUSCH with 2 physical resource blocks, we try to locate a PUSCH MCS index within 0 to 9 range (using QPSK), which can provide the closest coding rate in comparison with PSDCH. In order to select the desired MCS value, we first check different coding rates associated with different MCSs. Table 3 shows a transport block size table based on the Physical Resource Block (PRB) size and modulation and 
coding scheme. Here, $I_{T B S}$ is the index that can be used to map to the MCS scheme and $N_{P R B}$ is the physical resource block size (e.g., 1, 2, .., 10). The coding rate for PUSCH reference channel A3-5 (QPSK) [5] with 2 resource blocks and MCS 8 can be derived by

$$
\begin{gathered}
C R_{A 3-5}=\frac{N_{T B}+N_{C R C}}{N_{R B} \times N_{S C}^{R B} \times N_{\text {sym }}^{S C} \times N_{\text {bits }}^{\text {sym }}}=\frac{256+24}{2 \times 12 \times 12 \times 2} \\
=0.486
\end{gathered}
$$

Here, $C R_{A 3-5}$ is referred to as the coding rate of channel A3-5, while other notations are the same as Equation (1). Notice that $N_{s y m}^{S C}$ equals 12, because the last symbol of each subcarrier in the PUSCH is kept for transmission, and not punctured as it is done in the PSDCH. Since the reference channel A3-5 of PUSCH, with MCS equal to 8 and PRB equals 2, has the closest coding rate as PSDCH, its performance can be estimated directly using MATLAB LTE toolbox, and thus we use its simulation results to approximate PSDCH performance in our study.

\subsection{Physical Sidelink Shared Channel (PSSCH)}

The PSSCH is the data channel in D2D communication and its format (resource allocation, MCSs) is defined in the SCI transmitted by PSCCH as mentioned previously. Similar to the PSDCH, the channel structure of the PSSCH is extended based on the LTE PUSCH. Two main differences between PSSCH and PUSCH are summarized below.

First, similar to PSDCH, PSSCH uses physical layer broadcast solution, and no physical layer feedback exists. Thus, no link layer adaptation is used in the PSSCH, meaning that MCS cannot be changed once the PSSCH has been setup. Unlike PUSCH, the transmitter UE in PSSCH always transmits $4 \mathrm{RVs}$ of the transport block, regardless of the channel condition, and the $4 \mathrm{RVs}$ will be soft combined on the receiver UE to improve successful transmission. In addition, similar to the channel structure of other D2D physical channels, the last symbol of each subcarrier in a subframe is punctured to avoid the collision due to possible lack of accurate timing advance information in D2D communication. Second, the PSSCH only supports QPSK and 16 QAM modulations, which correspond to the modulation used in MCS 0 to 20 of LTE uplink shared channel.

Considering the minor differences between PSSCH and PUSCH, we again leverage the LTE MATLAB toolbox and modify the PUSCH configuration to simulate four PSSCH transmissions, for MCSs values between 0 and 20 .

\section{Performance Evaluation}

We have implemented the D2D channels described in Section 2 using the MATLAB LTE toolbox [3]. We now show the performance of these D2D channels. To measure the performance of D2D channels under different channel conditions, we consider BLER as the key performance metric. Recall that BLER is defined as the ratio of the number of erroneous blocks to the total number of blocks received through the simulated wireless channel. To simulate different channel conditions, we vary the SNR which is the ratio of desired signal strength to noise power.

To identify the relationship between BLER and SNR for the AWGN channel, additive white Gaussian noise is included in the transmitted signal. By varying the noise power, we simulate the signal transmission and reception to measure the BLER under different levels of SNR. To obtain the performance BLER versus SNR, we have used the Monte Carlo 
simulation. For each subframe, we have generated a transport block, and passed the transport block through the physical processing chains described in Section 3. At the receiver, CRC error checking is used to detect error in the received block. Each data point is the average value using 10,000 subframes to obtain sufficiently large sample data in order to compute the average BLER for a given SNR.

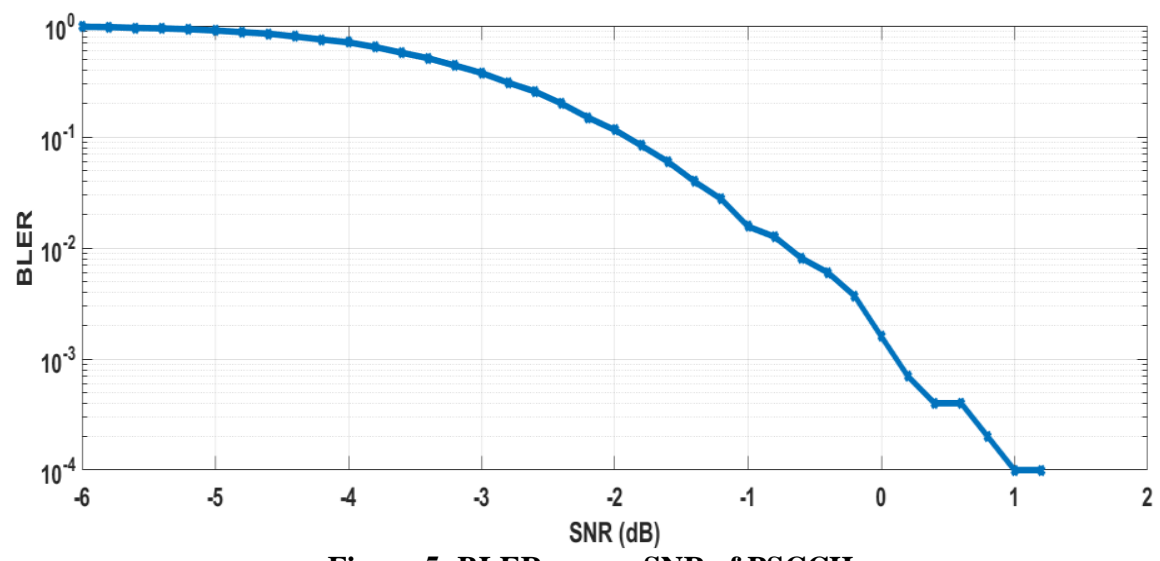

Figure 5: BLER versus SNR of PSCCH

Figure 5 illustrates the BLER versus SNR of one transmit instance for PSCCH. Recall that there are two identical instances for every transport block in PSCCH. As we can see in the figure, BLER decreases with the increase of SNR. For example, in order to keep BLER at $1 \%, \mathrm{SNR}$ is required to be around $-0.7 \mathrm{~dB}$. Figure 6 shows the performance of PSBCH with respect to BLER versus SNR. We have the similar observation. In this case, to achieve $1 \%$ error rate, SNR requires about $-6.2 \mathrm{~dB}$. PSBCH has better BLER performance than $\mathrm{PSSCH}$ as it provides more error protection. For the reference channels we used in our simulation, PSBCH has a coding rate of 0.056, while PSCCH has a coding rate of 0.22. Recall that, to increase the reliability of PSCCH, each transport block of PSCCH is transmitted in two identical instances, and these two instances are transmitted over two different subframes.

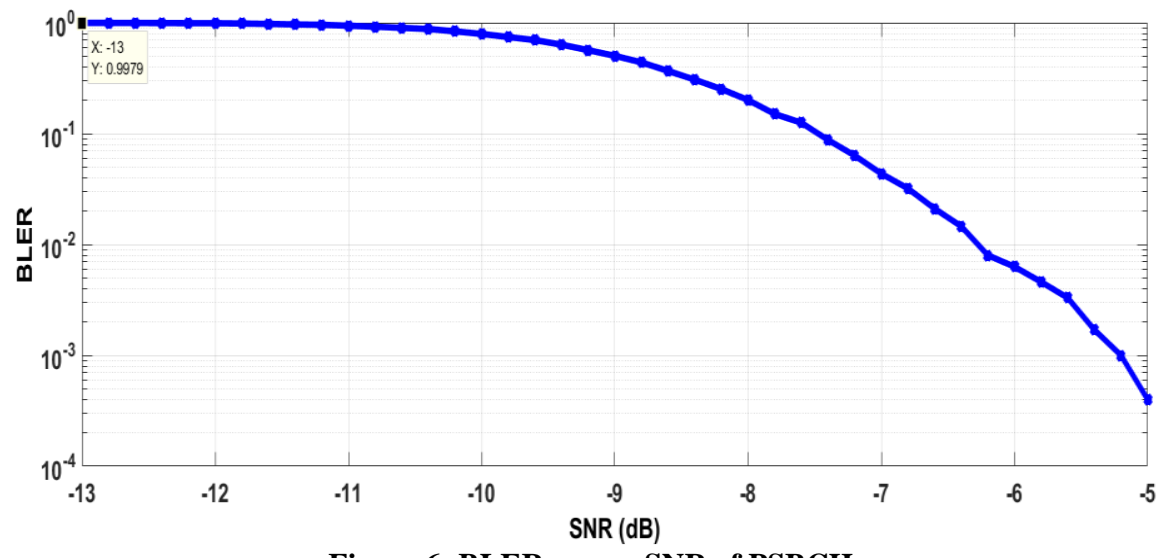

Figure 6: BLER versus SNR of PSBCH

For PSDCH, recall that, since no feedback mechanism exists at D2D physical channels, to increase the reliability of the channel, up to $4 \mathrm{RVs}$ of data will be transmitted to the receiver, even if the channel condition is good. Then, the receiver will soft combine the 
RVs of data to recover the transport block data. Figure 7 shows the performance of PSDCH in terms of BLER versus SNR. As we can see in the figure, when more RVs are received, BLER performance becomes better in terms of requiring lower SNR value to achieve a certain BLER. This is because each RV contributed to the decoding process via soft combining with previously received RVs. The more information that the receiver has, the better the decoding accuracy for a given SNR will be achieved.

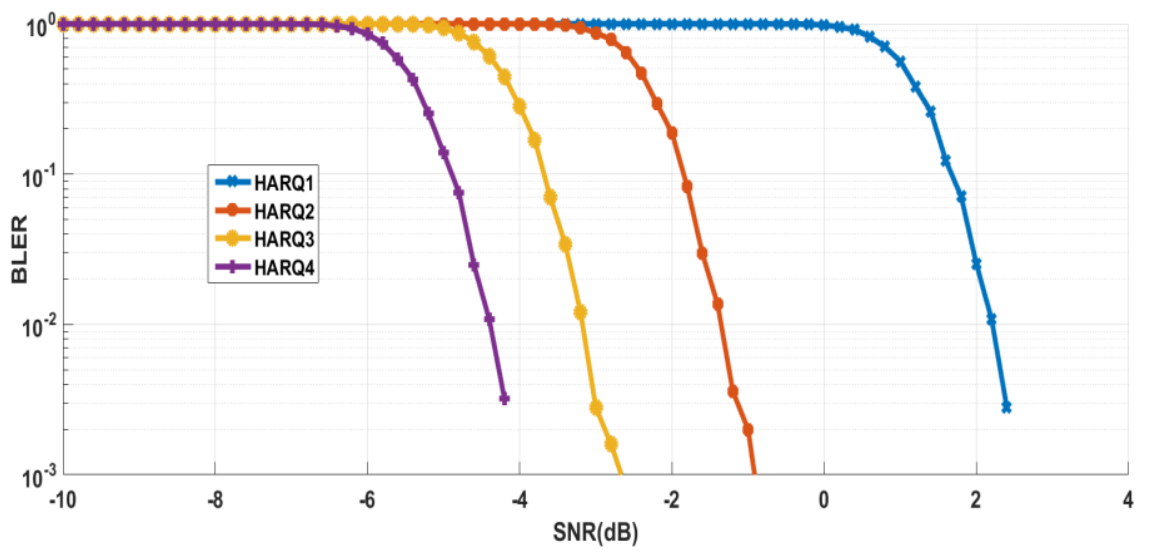

Figure 7: BLER and SNR of PSDCH

While the transport block on the PSDCH may be transmitted up to four times and use a fixed transport size, data on the PSSCH is always transmitted in 4 RV and supports MCS from 0 to 20. Also, notice that the transport size of PSSCH is determined by physical resource block size and its MCS. Figure 8(a)-(d) shows the performance of BLER versus SNR for MCS0-20 for four RVs, respectively. As we can see from these subfigures, when the number of HARQ retransmission increases, the better BLER performance can be achieved for a given SNR. Recall that in LTE D2D, HARQ corresponds to a RV of the data, which contains different combinations of information bits and error protection bits. In Figure 8, we can observe a relatively large performance gap between MCS10 and MCS11 across all 4 HARQs, which is due to the change of the modulation scheme between MCS10 and MCS11. QPSK is used for MCS0 to MCS10 and 16QAM is used for MCS11 up to MCS20. 


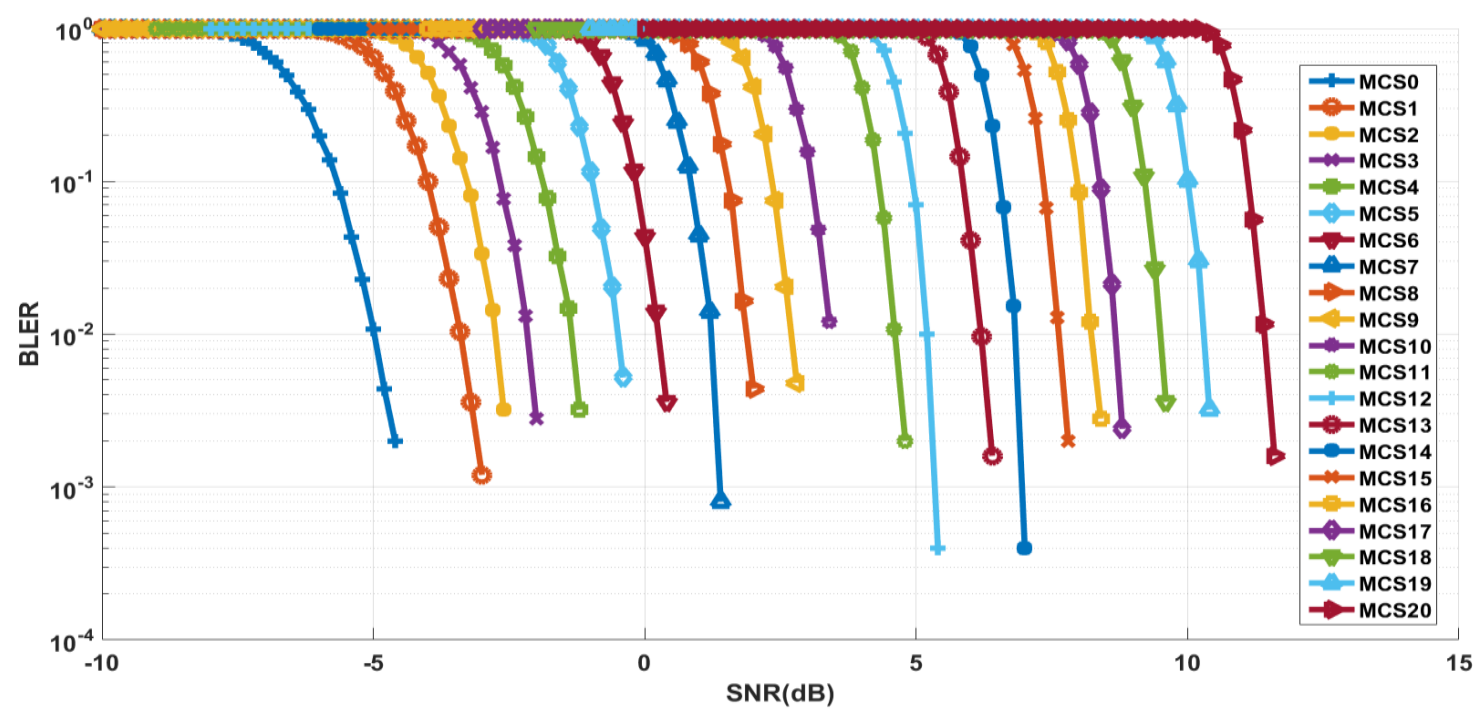

(a)

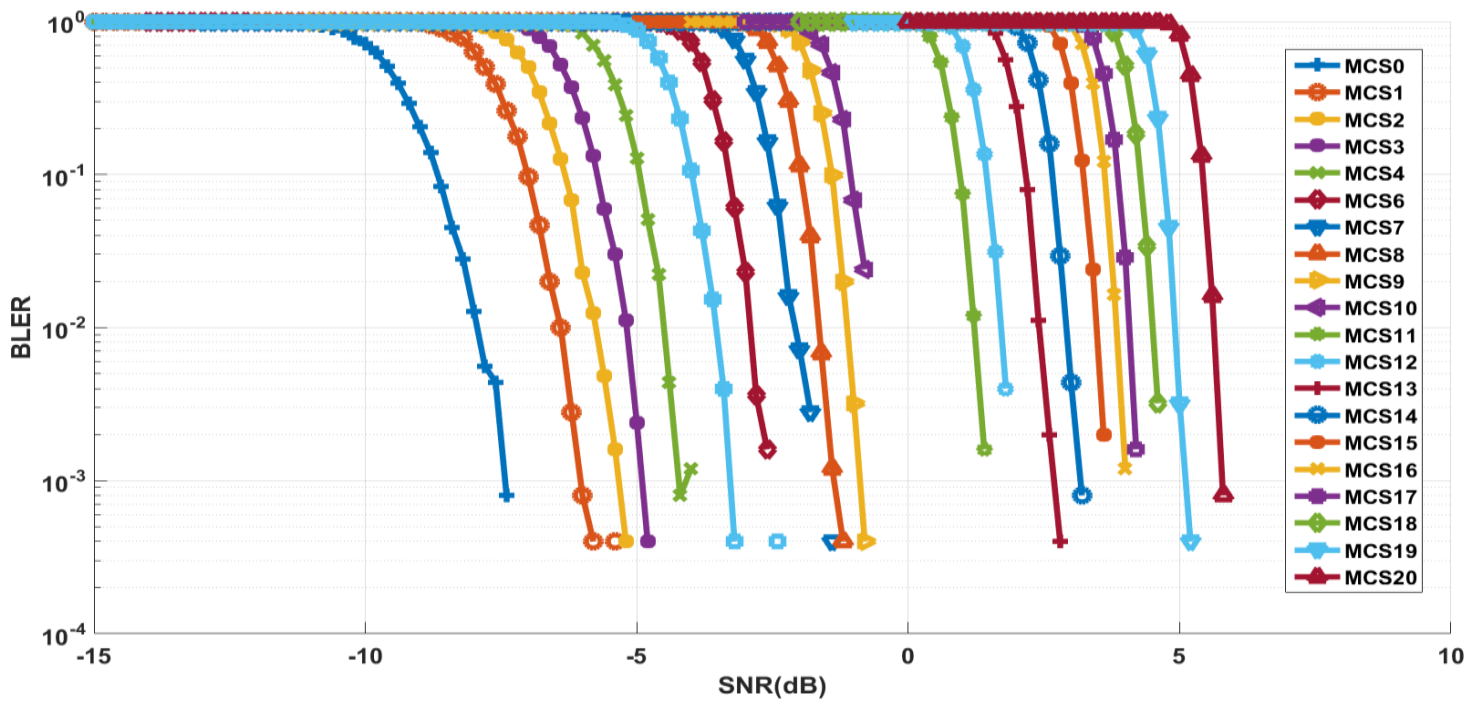

(b) 


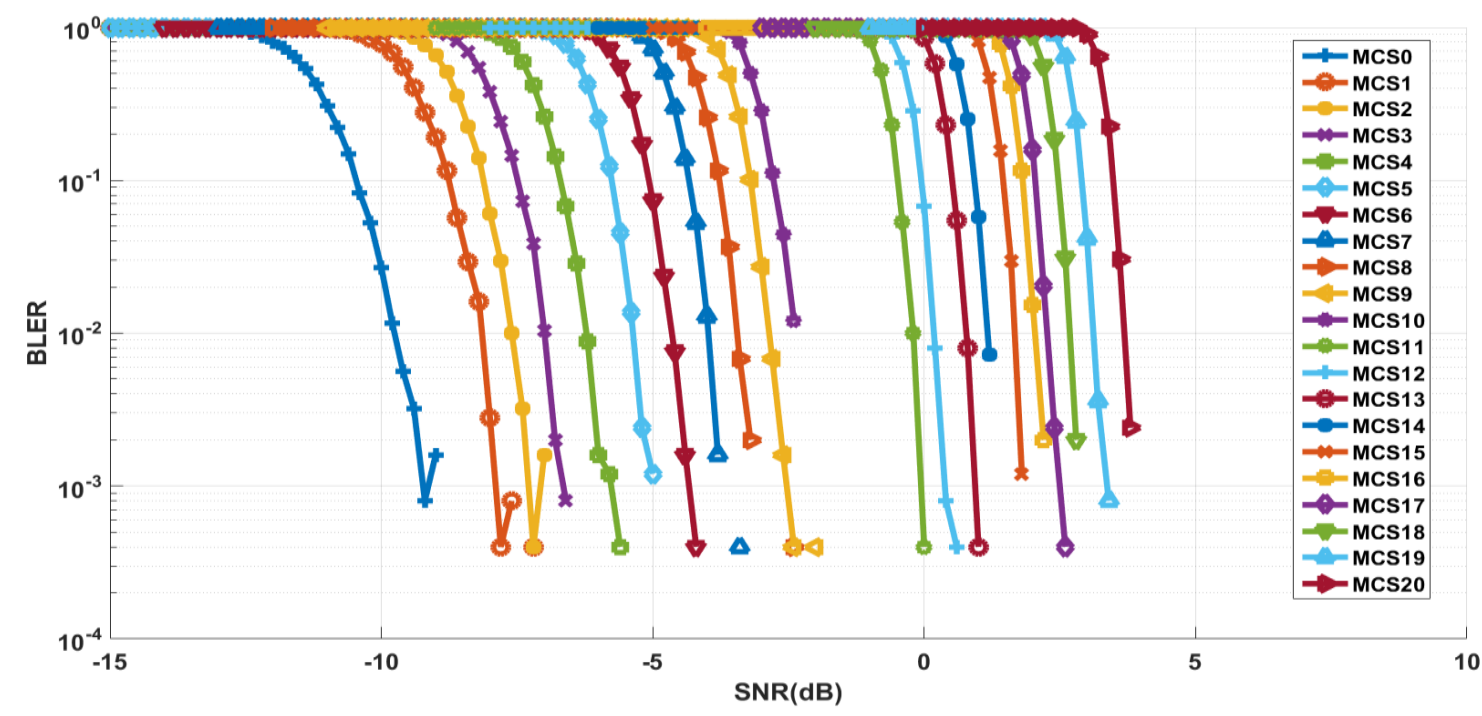

(c)

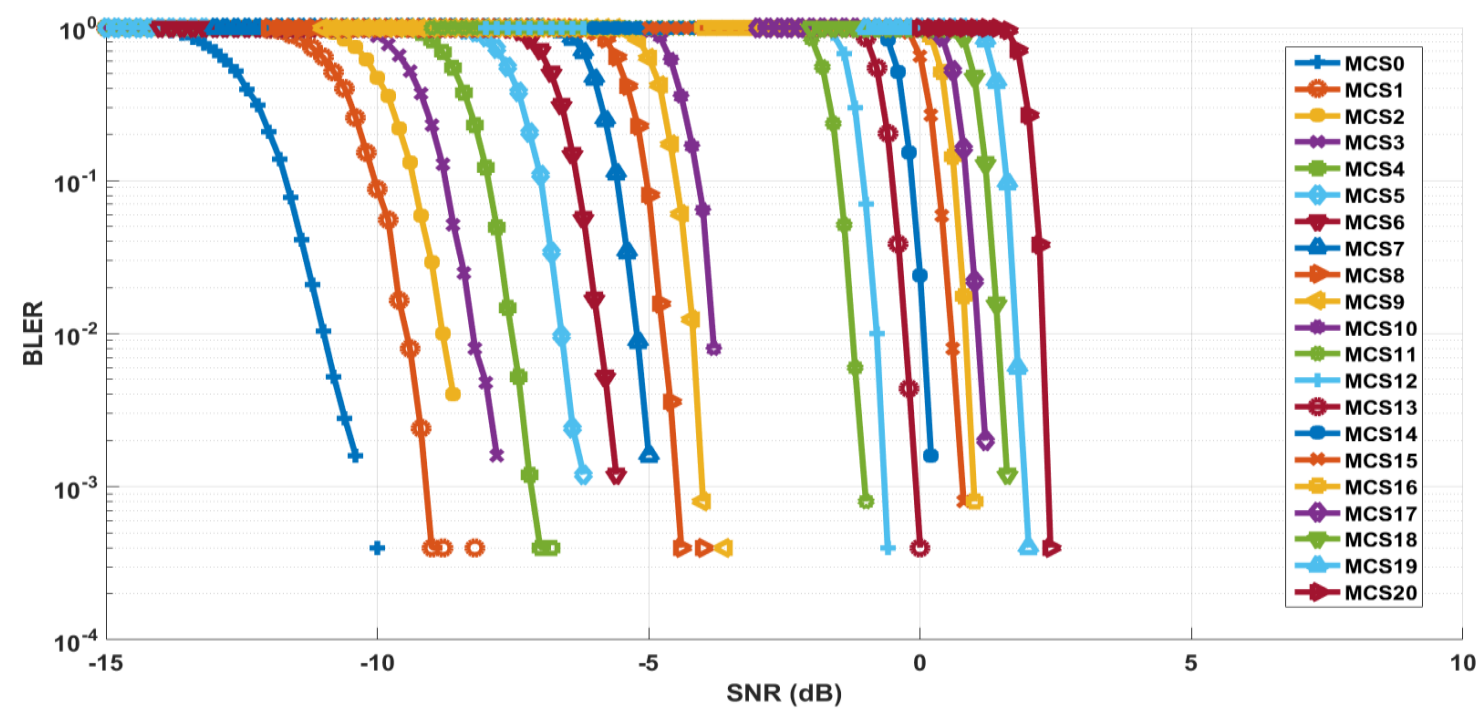

(d)

Figure 8: BLER versus SNR of PSSCH [(a): HARQ 1, (b): HARQ 2, (c): HARQ 3, (d) HARQ 4)]

\section{Incorporating D2D Physical Channel BLER Performance in Network Simulator}

Once we have the BLER performance data for D2D physical channels, the next step is to incorporate the physical layer simulation results into the network simulator to perform the system level simulation. The physical channel BLER performance is presented in a table with two columns, physical channel SNR and its corresponding BLER. A table lookup method is used in the system level simulator to find the BLER of the transport block based 
on the corresponding SINR. In ns-3, with the consideration of UEs' transmit power, path loss model, and small-scale fading model, we can obtain the SINR of every resource block. If a transport block consists of multiple resource blocks, by averaging the SINR of each resource block, we can derive the SINR of the transport block. Notice that, in our system level simulation, for the sake of simplicity, we treat the in-band interference from other UEs as AWGN noise. Therefore, SNR is used to approximate SINR.

In order to simulate whether a transport block can be received correctly in the system level simulation, a random variable that is uniformly distributed between 0 and 1 is generated and compared with the BLER value obtained via the table lookup. If the random value is smaller than BLER, the transmission of the transport block will be considered a failure, otherwise the transmission will be considered a success.

For the D2D physical channels using HARQ (e.g. PSDCH and PSSCH), to estimate BLER for a transmission, we need to consider not only the current SNR, but also the SNRs of previous HARQs, in order to obtain the effective SNR of the current transmission. Recall that a D2D transport block of the PSSCH is always transmitted using four HARQs because no physical layer feedback is available. Also, we have developed four BLER curves, where each BLER curve is associated with a HARQ transmission. Because the receiver combines the previous HARQ transmissions to decode the transport block, the later HARQ will achieve better BLER performance than previous HARQ transmissions. For example, as shown in Figure 9, HARQ ${ }_{1}$ represents the BLER curve of the first HARQ, and HARQ 2 shows the BLER curve of the second HARQ. In this figure, the X-axis is the SNR in dB and the $\mathrm{Y}$-axis is the BLER value, which is a number between 0 and 1 . In order to derive the effective $S N R$ for $\mathrm{HARQ}_{2}$, we design an algorithm to combine the SNRs of $\mathrm{HARQ}_{1}$ and $\mathrm{HARQ}_{2}$.

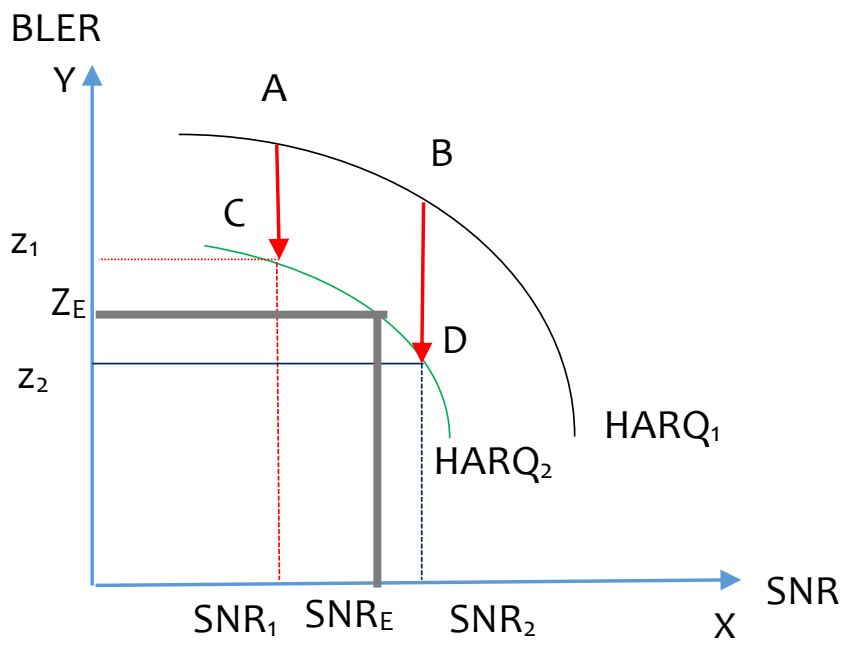

Figure 9: Combining two HARQ transmissions to obtain the effective SNR

Using Figure 9 as an example, we show the following two cases in our algorithm:

- In the first case (presented as A and D in Figure 9), $z_{1}$ is the BLER for HARQ 2 if both $\mathrm{HARQ}_{1}$ and HARQ 2 are transmitted at the $\mathrm{SNR}$ value of $\mathrm{SNR}_{1}$, and $\mathrm{z}_{2}$ is the BLER for $\mathrm{HARQ}_{2}$ if the $\mathrm{SNR}$ of both transmissions are $\mathrm{SNR}_{2}$. Since our effective $\mathrm{SNR}$ is a value between $\mathrm{SNR}_{1}$ and $\mathrm{SNR}_{2}$, intuitively, its corresponding BLER, $\mathrm{Z}_{\mathrm{E}}$, will be a value between $z_{1}$ and $z_{2}$. Then, we apply the weighted averaging 
mechanism to approximate BLER of $\mathrm{HARQ}_{2}$ and give more recent HARQ higher weight using the following equation,

$$
Z_{E}=\frac{x_{1} z_{1}+x_{2} z_{2}}{x_{1}+x_{2}}=\frac{z_{1}+\alpha z_{2}}{1+\alpha}
$$

Here, $Z_{E}$ is the effective BLER of the $\mathrm{HARQ}_{2}, x_{1}$ and $x_{2}$ are $\mathrm{SNR}_{1}$ and $\mathrm{SNR}_{2}$ in the linear scale, respectively, where $x_{1}=10^{S N R_{1} / 10}$ and $x_{2}=10^{S N R_{2} / 10}$, and $\alpha=\frac{x_{2}}{x_{1}}=$ $10^{\left(S N R_{2}-S N R_{1}\right) / 10}$.

- In the second case (presented as B and C in Figure 9), if the $S N R$ of HARQ 1 is $\mathrm{SNR}_{2}$ and the $\mathrm{SNR}$ of $\mathrm{HARQ}_{2}$ is $\mathrm{SNR}_{1}$. Similarly, we use the following equation to estimate BLER for $\mathrm{HARQ}_{2}$, and again more recent transmission $\mathrm{HARQ}_{2}$ is given higher weight.

$$
Z_{E}=\frac{x_{2} z_{1}+x_{1} z_{2}}{x_{1}+x_{2}}=\frac{z_{2}+\alpha z_{1}}{1+\alpha}
$$

Here, $Z_{E}$ is the effective $\mathrm{SNR}$ of the $\mathrm{HARQ}_{2}, x_{1}$ and $x_{2}$ are $\mathrm{SNR}_{1}$ and $\mathrm{SNR}_{2}$ in the linear scale, respectively, where $x_{1}=10^{S N R_{1} / 10}$ and $x_{2}=10^{S N R_{2} / 10}$, and $\alpha=\frac{x_{2}}{x_{1}}=$ $10^{\left(S N R_{2}-S N R_{1}\right) / 10}$.

By using this algorithm, we can combine the information for the HARQ 1 and HARQ 2 so that an effective $\mathrm{SNR}$ for $\mathrm{HARQ}_{2}$ and its corresponding BLER, $\mathrm{Z}_{\mathrm{E}}$, can be derived. In the similar manner, this algorithm can be continually used to combine the results of $\mathrm{HARQ}_{2}$ and $\mathrm{HARQ}_{3}$ so that the effective $\mathrm{SNR}$ for $\mathrm{HARQ}_{3}$ can be derived, and so on.

\section{Final Remarks}

In this study, we have investigated four types of D2D physical channels, i.e. PSCCH, PSBCH, PSDCH, and PSSCH. We have described those channels and the implementation details based on the MATLAB LTE toolbox. Via an extensive simulation study, we have showed the BLER performance of those channels with respect to the SNR. The results of this work have been wrapped in a D2D error model for LTE-based D2D system level simulation.

\section{References}

[1] 3GPP TS 23.303 V12.7.0, "Technical Specification Group Services and System Aspects: Proximity-based Services (ProSe): Stage 2 Release 12,” 2015.

[2] Arash Asadi and Qing Wang, "A Survey on Device-to-Device Communication in Cellular Networks," IEEE Communications Surveys \& Tutorials, vol. 16, no. 4, pp: 18011819, November 2014.

[3] LTE System Toolbox, http://www.mathworks.com/products/lte-system/.

[4] LTE Modules, https://www.nsnam.org/docs/models/html/lte.html.

[5] 3GPP TS 36.101 V14.0.0, "Evolved Universal Terrestrial Radio Access (E-UTRA); User Equipment (UE) Radio Transmission and Reception,” July 2016. 
[6] 3GPP TS 36.212 V13.2.0, "Evolved Universal Terrestrial Radio Access (E-UTRA); Multiplexing and Channel Coding," June 2016.

[7] 3GPP TS 36.211 V13.2.0, "Evolved Universal Terrestrial Radio Access (E-UTRA); Physical Channels and Modulation,” July 2016.

[8] 3GPP TS 36.213 V13.2.0, "Evolved Universal Terrestrial Radio Access (E-UTRA); Physical Layer Procedures,” June 2016. 